\title{
Structure of Surfaces and Interfaces as Studied Using Synchrotron Radiation. Liquid Surfaces
}

\section{Citation}

Pershan, Peter S. 1990. Structure of surfaces and interfaces as studied using synchrotron radiation. Liquid surfaces. Faraday Discussions of the Chemical Society 89:231-245.

\section{Published Version}

doi:10.1039/dc9908900231

\section{Permanent link}

http://nrs.harvard.edu/urn-3:HUL.InstRepos:10357350

\section{Terms of Use}

This article was downloaded from Harvard University's DASH repository, and is made available under the terms and conditions applicable to Other Posted Material, as set forth at http:// nrs.harvard.edu/urn-3:HUL.InstRepos:dash.current.terms-of-use\#LAA

\section{Share Your Story}

The Harvard community has made this article openly available.

Please share how this access benefits you. Submit a story.

\section{Accessibility}




\title{
Structure of Surfaces and Interfaces as studied using Synchrotron Radiation
}

\author{
Liquid Surfaces \\ P. S. Pershan \\ Physics Department and Division of Applied Science, Harvard University, Cambridge, \\ MA 02138, USA
}

The use of specular reflection of X-rays to study the structure of the liquid/vapour interfaces along the direction normal to the surface is described. If $R_{\mathrm{F}}(\theta)$ is the theoretical Fresnel reflection law for $\mathrm{X}$-rays incident on an ideal flat surface at an angle $\theta$, and $R(\theta)$ is the measured reflectivity from the true surface, the ratio $R(\theta) / R_{F}(\theta)$ is a measure of the electron density along the surface normal; i.e.

$$
\frac{R(\theta)}{R_{\mathrm{F}}(\theta)} \approx\left|\frac{1}{\rho_{\infty}} \int \frac{\partial\langle\rho(z)\rangle}{\partial z} \exp \left(\mathrm{i} Q_{z} z\right) \mathrm{d} z\right|^{2}
$$

where $\rho_{\infty}$ is the electron density far from the surface, $\partial\langle\rho(z)\rangle / \partial z$ is the gradient of the average electron density along the surface normal and $Q_{z}=(4 \pi / \lambda) \sin (\theta)$. For simple liquids $\rho_{\infty}^{-1} \partial\langle\rho\rangle / \partial z \approx$ $\left[1 / \sqrt{ }\left(2 \pi \sigma^{2}\right)\right] \exp \left(-z^{2} / 2 \sigma^{2}\right)$, and $R(\theta) / R_{\mathrm{F}}(\theta) \approx \exp \left(-Q^{2} \sigma^{2}\right)$, where $\sigma^{2}$ is dominated by the mean-square average of thermally excited fluctuations in the height of the surface. For liquid crystals and for lyotropic micellar systems temperature-dependent structure in $R(\theta)$ is due to surface-induced layering in $\langle\rho(z)\rangle$. Other experimental results from thin layers of liquid ${ }^{4} \mathrm{He}$ and monolayers, of amphipathic molecules on the surface of $\mathrm{H}_{2} \mathrm{O}$ will be described. The possibility of complementing specular reflectivity measurements of surface roughness by studying diffuse scattering at small angles off of the specular condition will also be illustrated with results from the $\mathrm{H}_{2} \mathrm{O}$ surface.

Although X-ray specular reflection from surfaces was observed over sixty years ago ${ }^{1-4}$ practical application to the characterization of surfaces has only been done recently. The purpose of this manuscript is to review some of the fundamental principles of the technique and to report some of our recent results on liquid and solid surfaces. In addition we will also discuss related experiments in which diffuse scattering is observed at small angles from the specular condition. The latter can be used to study structure within the plane of the surface.

The basic idea for specular reflectivity is the recognition that even at X-ray wavelengths one can introduce a macroscopic dielectric constant to describe the average properties of the electromagnetic waves in materials:

$$
\varepsilon \approx 1-4 \pi \rho e^{2} / m \omega^{2}=1-\rho r_{\mathrm{e}} \lambda^{2} / \pi
$$

where $\rho$ is the electron density in the material, $\lambda$ is the $X$-ray wavelength (i.e. $\lambda \omega / 2 \pi=c$ ), $m$ and $e$ are the electron mass and charge, respectively, and $r_{\mathrm{e}}$ is the classical radius of the electron. $\dagger$ Neglecting polarization effects, which are not significant at small angles,

\footnotetext{
† This form neglects both absorption and dispersion. The effect of dispersion can be accounted for if the electron density $\rho=(1 / V) \sum_{i} Z_{j}$ is replaced by $\rho^{e 4}=(1 / V) \sum_{j} f_{i}(0)$, where $Z_{j}$ is the number of electrons on the $j$ th atom, $f_{j}(0)$ is the real part of the atomic scattering factor of the $j$ th atom in the forward direction, and the sum $\{j\}$ includes all atoms in the volume $V$. For most cases $\rho \approx \rho^{\mathrm{e}}$. The effect of absorption is included by setting $\varepsilon^{\prime \prime}=(\lambda / 2 \pi) \mu$, where $\mu^{-1}$ is the X-ray decay length of the material for power.
} 
the classical result for the reflection coefficient of an electromagnetic wave incident at an angle $\theta$ (i.e. $\theta=0$ is parallel to the surface) from an ideal flat interface between vacuum $(\varepsilon=1)$ and material of relative permittivity $\varepsilon$ is:

$$
R_{\mathrm{F}}(\theta)=\left|\frac{\sin (\theta)-\sqrt{ }\left[\varepsilon-\cos ^{2}(\theta)\right]}{\sin (\theta)+\sqrt{ }\left[\varepsilon-\cos ^{2}(\theta)\right]}\right|^{2}
$$

There is a critical angle $\theta_{\mathrm{c}}=\cos ^{-1}(\varepsilon) \approx \sqrt{ }\left(\rho r_{\mathrm{e}} \lambda^{2} / \pi\right)$ such that for $\theta \leqslant \theta_{\mathrm{c}}, R_{\mathrm{F}}(\theta)=1$ and for $\theta \gg \theta_{\mathrm{c}}, R_{\mathrm{F}}(\theta) \approx\left(\theta_{\mathrm{c}} / 2 \theta\right)$. $^{4}$ For water, $\theta_{\mathrm{c}}=0.152^{\circ}$ when $\lambda=1.54 \AA$.

The mechanism for characterization of the structure of non-ideal surfaces, along the normal direction, depends on analysis of deviations between the measured reflectivity $R(\theta)$ and the ideal, or Fresnel, reflectivity $R_{\mathrm{F}}(\theta)$ over a range of angles $\theta \sim \lambda /(2 \Delta L)$, where $\Delta L$ is one measure of the spatial resolution. Since $R_{\mathrm{F}}(\theta)$ falls as the fourth power of the incident angle, meaningful chacterization of many surfaces require measurements over a very wide dynamic range of reflectivities, typically $10^{(9-10)}$, and this is the primary reason why synchrotron radiation is required.

\section{Theory for Non-ideal Surfaces}

\section{Specular Reflectivity}

When $\theta \gg \theta_{c}$ the reflection from a real surface is most easily obtained by summing over the scattering from infinitesimally thin layers $\delta z$ at some distance $z$ from the average location of the interface, which is taken to be the $x-y$ plane at $z=0$. For incident wavevector $\boldsymbol{k}^{0}$ the amplitude of the scattered wave at a distance $R$ from the sample, in a direction defined by $\boldsymbol{k}^{\mathrm{s}}$, is approximately given by

$$
\frac{\delta E_{\mathrm{s}}}{E_{0}} \approx r_{\mathrm{e}} \delta z\left\{\frac{\exp [-\mathrm{i}(\omega R / c)]}{R}\right\} \int \mathrm{d} x \mathrm{~d} y \rho(x, y, z) \exp \left\{\mathrm{i}\left[\boldsymbol{k}^{\mathrm{s}}-\boldsymbol{k}^{0}\right] \cdot \boldsymbol{r}\right\} .
$$

Setting $\rho(x, y, z)=\langle\rho(z)\rangle+[\rho(x, y, z)-\langle\rho(z)\rangle]$, where $\langle\rho(z)\rangle$ is the average of $\rho(x, y, z)$ over an $x-y$ plane at height $z$, the first term in the integral in eqn (3) obtains

$$
\frac{\delta E_{\mathrm{s}}}{E_{0}} \approx r_{\mathrm{e}} \delta z \frac{\langle\rho(z)\rangle \exp [-\mathrm{i}(\omega R / c)]}{R}\left[4 \pi^{2} \delta\left(Q_{x}\right) \delta\left(Q_{y}\right)\right] \exp \left(\mathrm{i} Q_{z} z\right)
$$

where $\boldsymbol{Q} \equiv \boldsymbol{k}^{\mathrm{s}}-\boldsymbol{k}^{0}$. Since the condition that $Q_{x}=Q_{y}=0$ corresponds to the incident and reflected angles being equal and in the same plane, this term can be identified with the contribution of the thin layer to the specularly reflected signal. The term proportional to $[\rho(x, y, z)-\langle\rho(z)\rangle]$ gives rise to diffuse scattering that will be discussed below.

When $\theta \gg \theta_{c}$ a useful expression for the ratio of the specularly reflected signal to that from an ideal surface,

$$
\frac{R(\theta)}{R_{\mathrm{F}}(\theta)} \approx\left|\frac{1}{\rho} \int \mathrm{d} z \frac{\mathrm{d}\langle\rho(z)\rangle}{\mathrm{d} z} \exp \left(\mathrm{i} Q_{z} z\right)\right|^{2}
$$

is obtained by the following steps: ${ }^{6.7}$ (1) Integrate eqn (4) from $z=-\infty$ to $+\infty$ by parts to express the answer in terms of $\mathrm{d}\langle\rho(z)\rangle / \mathrm{d} z$. (2) Square the result and use of the standard interpretation to substitute $\left[4 \pi^{2} \delta\left(Q_{x}\right) \delta\left(Q_{y}\right)\right]^{2}=A_{x y}\left[4 \pi^{2} \delta\left(Q_{x}\right) \delta\left(Q_{y}\right)\right]$, where $A_{x y}$ is the illuminated cross-sectional area of the interface. (3) Calculate the detected power by integrating $c\left|E_{\mathrm{s}}\right|^{2} / 4 \pi$ over the area of the detector. Since the solid angle of the detector can be expressed as, $\mathrm{d} \Omega=(\lambda / 2 \pi)^{2}(1 / \theta) \mathrm{d} Q_{x} \mathrm{~d} Q_{y}$, this eliminates the $\delta$ functions. (4) Normalize the scattered intensity to the incident power $\theta A_{x y} c\left|E_{0}\right|^{2} / 4 \pi$. Eqn (5) follows if one uses the asymptotic form $R_{\mathrm{F}}(\theta) \approx\left(\theta_{\mathrm{c}} / 2 \theta\right)^{4}$ with $Q_{z} \approx(4 \pi / \lambda) \theta$.

There are a large class of problems, some of which will be illustrated below, for which $R(\theta) / R_{\mathrm{F}}(\theta) \approx 1$ for small $\theta$. In these cases eqn (5) can be used for all $\theta$ so long 
as $Q_{z}$ corresponds to the value inside the material; i.e. $Q_{z} \approx(4 \pi / \lambda) \sqrt{ }\left(\theta^{2}-\theta_{\mathrm{c}}^{2}\right)$. A different approach, that allows for solutions when $R(\theta) / R_{\mathrm{F}}(\theta) \neq 1$ for small $\theta$ is to solve the one-dimensional wave-equation

$$
\frac{\mathrm{d}^{2} E}{\mathrm{~d} z^{2}}+\frac{1}{4}\left(Q_{z}^{2}-Q_{\mathrm{c}}^{2} \frac{\langle\rho(z)\rangle}{\rho}\right) E=0
$$

where $Q_{\mathrm{c}} \equiv(4 \pi / \lambda) \theta_{\mathrm{c}}$, with suitable boundary conditions. For $\theta \gg \theta_{\mathrm{c}}$ the results are identical to those of eqn (5) regardless of whether or not $R(\theta) \neq R_{\mathrm{R}}(\theta)$ for small $\theta$.

\section{Diffuse Scattering}

Diffuse scattering is observed when the wavevector difference $\boldsymbol{Q}$ has a non-vanishing component $q \equiv\left(Q_{x}, Q_{y}\right)$ parallel to the surface. For an incident angle $\theta$, and detected radiation that makes angles $\theta^{\prime}$ with the surface and $\Psi$ with the plane of incidence

$$
\begin{aligned}
& Q_{x}=(2 \pi / \lambda) \cos \left(\theta^{\prime}\right) \sin (\psi) \\
& Q_{y}=(2 \pi / \lambda)\left[\cos (\theta)-\cos \left(\theta^{\prime}\right) \cos (\psi)\right] \approx(2 \pi / \lambda)\left[1-\theta^{2} / 2-\left(1-\theta^{\prime 2} / 2\right) \cos (\psi)\right] \\
& Q_{z}=(2 \pi / \lambda)\left[\sin \left(\theta^{\prime}\right)+\sin (\theta)\right] \approx(2 \pi / \lambda)\left[\theta^{\prime}+\theta\right] .
\end{aligned}
$$

If the mean-square variation in the height of the surface inhomogeneities is small compared to $1 / Q_{z}$ one can define a surface density $\rho_{\mathrm{s}}(x, y)$ and make the approximation, $[\rho(x, y, z)-\langle\rho(z)\rangle] \approx \rho_{\mathrm{s}}(x, y) \delta(z)$. The differential cross-section for surface diffuse scattering can then be expressed as

$$
\frac{\mathrm{d} \sigma}{\mathrm{d} \Omega}=\left(r_{\mathrm{e}}^{2}\right) A_{x y} T(\theta) T\left(\theta^{\prime}\right) \int \mathrm{d} x \mathrm{~d} y\left\langle\rho_{\mathrm{s}} \rho_{\mathrm{s}}(x, y)\right\rangle \exp (\mathrm{i} \boldsymbol{q} \cdot \boldsymbol{r})
$$

where 8,9

$$
T(\theta)=\left(2 \theta / \theta_{\mathrm{c}}\right)^{2} \sqrt{ }\left[R_{\mathrm{F}}(\theta)\right]
$$

The surface enhancement factor $T(\theta)$ occurs because scattering from surface inhomogeneities is proportional to the square of the total surface field, not the square of the incident field. For $\theta \leqslant \theta_{\mathrm{c}}$ the amplitudes of the reflected and incident fields are equal and because the phase of the reflected wave varies from $\pi$, when $\theta \approx 0$, to 0 when $\theta \approx \theta_{\mathrm{c}}$, the total field at the surface varies from zero to twice the incident field for $\theta \approx \theta_{\mathrm{c}}$. The function $T(\theta)$, which is proportional to the square of the field, varies from 0 to 4 , as $\theta$ increases from 0 to $\theta_{\mathrm{c}}$, and then falls to unity for $\theta \gg \theta_{\mathrm{c}}$. The factor $T\left(\theta^{\prime}\right)$ appears because of a similar effect in the coupling between the surface currents and the scattered fields, and the angular dependence of these two factors helps in distinguishing surface scattering from other diffuse scattering processes. ${ }^{10}$ The cross-section for diffuse scattering can be expressed in term of height fluctuations $\eta(x, y)$ of the surface by the substitution $\left\langle\rho_{\mathrm{s}} \rho_{\mathrm{s}}(x, y)\right\rangle=\rho^{2}\langle\eta \eta(x, y)\rangle$ into eqn (8).

\section{Experimental}

The main features of the experimental geometry for studying the liquid/vapour interface are illustrated in fig. $1 .^{11,12}$ The incident beam is deflected downward by an angle $\theta$ and a detector, of height $h$ and width $w$ is located a distance $L$ from the sample surface. In order to insure that the incident beam strikes the centre of the sample for all $\theta$, it is on an elevator such that its vertical position can be continuously adjusted. The detector can be moved both vertically, to vary $\theta^{\prime}$, and along an arc to vary $\psi$. In all of our experiments the incident beam is highly collimated such that the spread in incident wavevector $\boldsymbol{k}^{0}$ is negligible. The height of the incident beam $h^{0}$, however, is not negligible 


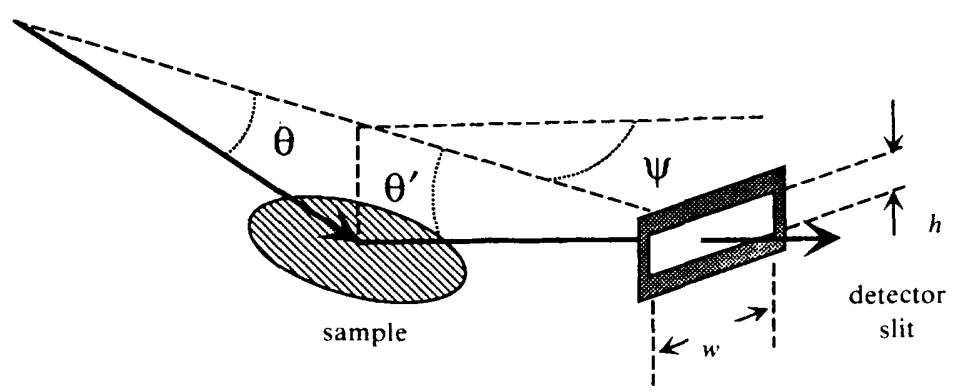

Fig. 1. Schematic illustration of the geometry for X-ray scattering study the liquid/vapour interface. The detector slit, of height $h$ and width $w$, is shown in the position for detection of the specularly reflected beam $\left(\theta=\theta^{\prime}\right.$ and $\left.\psi=0\right)$.

and for small angles $\theta$ the 'footprint' of the beam on the horizontal interface can be large. Verification of the alignment of beam and sample positions is achieved by measuring the specularly reflected intensity as a function of detector angle $\theta^{\prime}$, for different vertical displacements of the sample. Both the intensity and shape of the signal are constant for the range of sample positions where the beam is intercepted by a flat portion of the sample.

The height $h$ and the width $w$ of the detector slit are set in order to intercept all of the specularly reflected signal fully. When the surface is sufficiently flat this is identical to the physical size of the incident beam $\boldsymbol{k}^{0}$ at the detector position. For specular reflection studies the angular resolution of the spectrometer, $\Delta \theta$, is determined by a convolution of the angular distribution of the incident beam and the surface normal. In contrast, for diffuse scattering measurements and practical slit dimensions, the appropriate resolution is determined by a convolution of the detector size with a suitable projection of the illuminated cross-sectional area of the sample. If the height $h$ of the detector is larger than the height of the incident beam, and if the spectrometer is near to the specular condition $\left(\theta^{\prime} \approx \theta\right.$ and $\left.\psi \approx 0\right)$ the projection of the resolution (for diffuse scattering) on the horizontal liquid surface has full widths.

$$
\begin{aligned}
& \Delta Q_{y} \approx(2 \pi / \lambda) \theta^{\prime} h / L \\
& \Delta Q_{x} \approx(2 \pi / \lambda) w / L
\end{aligned}
$$

where $L$ is the distance from sample to detector.

The usual situation on scanning either $\theta^{\prime}$ or $\psi$ is that the measured intensity $I\left(\theta^{\prime}, \psi\right)$ has a sharp central peak at the specular condition and a broad flat background off of the specular. Specular reflection $R(\theta)$ is then taken to be the difference between the signal in the specular position and the diffuse scattering background at small values of either $\theta^{\prime}-\theta$ or $\psi$. For some surfaces the background depends on the offset and it is necessary to develop an extrapolation procedure suitable to the particular surface. ${ }^{13}$

\section{Results}

\section{Water}

\section{Specular Reflectivity/Roughness}

Specular reflectivity data from the free surface of $\mathrm{H}_{2} \mathrm{O}$, as shown in fig. 2(a), is typical of the reflectivity from a number of surfaces. ${ }^{8}$ There is a small region for $\theta<\theta_{c}$ where the reflectivity is essentially $100 \%$ followed by a rapid fall, shown here over eight orders 

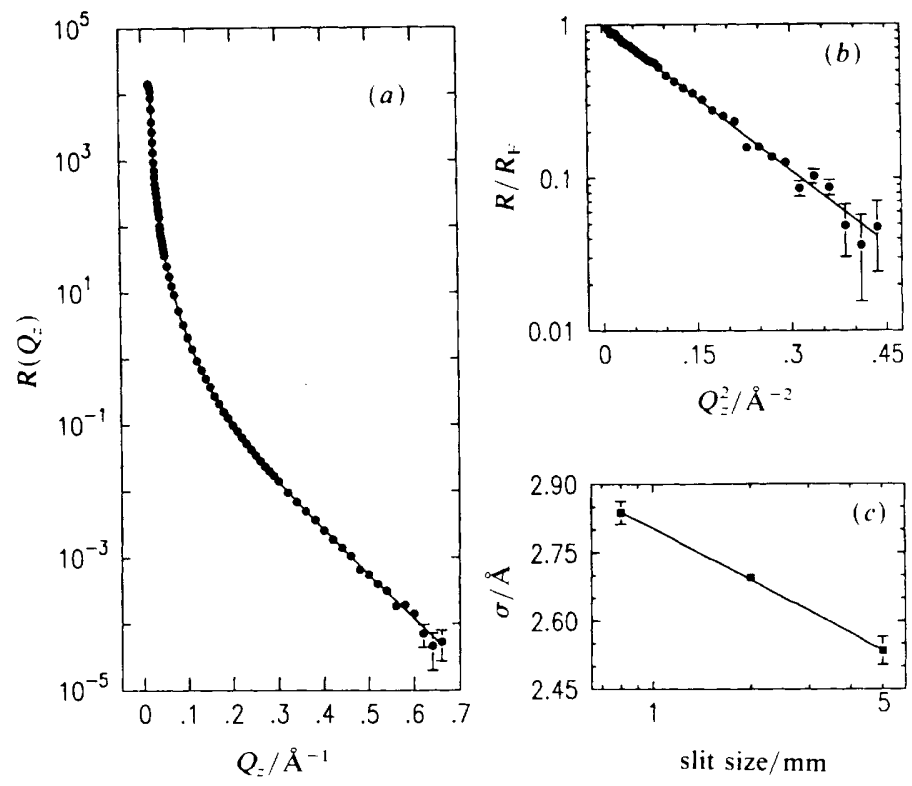

Fig. 2. (a) Measured reflectivity from $\mathrm{H}_{2} \mathrm{O}$ with a detector of height $h=2.0 \mathrm{~mm}$ at $600 \mathrm{~mm}$ from the sample. ${ }^{13}$ The solid line is the best fit of the theoretical form given by eqn (11) to the data. Error bars that are not shown are smaller than the size of the symbol. (b) The logarithm of the same data as in (a) plotted versus $Q_{z}^{2}$. The slope of the solid line indicates a value of $\sigma^{2}=$ $(2.70 \pm 0.03 \AA)^{2}$. (c) Comparison between the slope of data like that shown in $(b)$ for detector heights $h=0.8,20.0$ and $5.0 \mathrm{~mm}$ and the theoretical result for capillary wave roughness, eqn (15).

of magnitude. ${ }^{8,13,14}$ As can be seen from fig. $2(b)$, in which the same data are normalized to $R_{\mathrm{F}}(\theta)$ and plotted as $\log _{\mathrm{e}}\left[R(\theta) / R_{\mathrm{F}}(\theta)\right]$ versus $Q_{z}^{2}$, the data are essentially of the form

$$
\frac{R(\theta)}{R_{\mathrm{F}}(\theta)}=\exp \left(-Q_{=}^{2} \sigma^{2}\right)
$$

where $\sigma^{2}=(2.70 \pm 0.03 \AA)^{2}$. This data were taken from the surface of $\mathrm{H}_{2} \mathrm{O}$ on a Langmuir trough $^{15}$ in which the surface tension was monitored in situ to be $72.5 \pm 0.4 \mathrm{dyn} \mathrm{cm}{ }^{-1}$, the detector height was $2.0 \mathrm{~mm}$ and the distance to the sample was $c a .600 \mathrm{~mm} . \dagger$ It is straightforward to demonstrate that this form is consistent with the profile $\langle\rho(z)\rangle / \rho=$ $\left[1 / \sqrt{ }\left(2 \pi \sigma^{2}\right)\right] \exp \left(-z^{2} / 2 \sigma^{2}\right)$ by substitution into eqn (5). Alternatively, one can numerically integrate the data to obtain the Patterson function: ${ }^{16}$

$$
Z(s) \equiv \frac{1}{\pi} \operatorname{Re}\left\{\int_{0}^{+\infty} \frac{R(\theta)}{R_{\mathrm{F}}(\theta)} \exp \left(\mathrm{i} Q_{z} z\right) \mathrm{d} z\right\}=\frac{1}{\rho^{2}} \int_{-\infty}^{\infty}\left(\frac{\mathrm{d}\langle\rho(z)\rangle}{\mathrm{d} z}\right)\left(\frac{\mathrm{d}\langle\rho(s+z)\rangle}{\mathrm{d} z}\right) \mathrm{d} z
$$

with $Q_{z}=(4 \pi / \lambda) \sin (\theta)$, and demonstrate directly that

$$
Z(s) \approx\left[1 / \sqrt{ }\left(4 \pi \sigma^{2}\right)\right] \exp \left(-s^{2} / 4 \sigma^{2}\right) .
$$

It is interesting to compare this measurement with the reflection predicted by assuming the water surface is made rough by thermal excitation of capillary waves. ${ }^{8.14}$ Taking $\gamma$

+ A previously reported larger value of $\sigma^{2}=(3.3 \pm 0.1 \AA)$ was reported for a $\mathrm{H}_{2} \mathrm{O}$ surface in which the surfaced tension was not monitored; ${ }^{*}$ however, an independent measurement on a clean surface essentially agrees with the present value. ${ }^{14}$ 
to be the surface tension and $\eta(x, y)$ to be the height of the water surface at some point $(x, y)$ the energy per unit area of a rough surface is given by:

$$
U=\frac{\gamma}{2}\left[\left(\nabla_{x} \eta\right)^{2}+\left(\nabla_{y} \eta\right)^{2}\right]+\frac{\rho g}{2} \eta(x, y)^{2}
$$

where $g$ is the acceleration of gravity. From standard statistical physics the mean square value of the height fluctuations:

$$
\left\langle\eta(x, y)^{2}\right\rangle=\frac{k_{\mathrm{B}} T}{4 \pi^{2} \gamma} \int_{0}^{Q_{\max }} \mathrm{d} Q_{x} \mathrm{~d} Q_{y} \frac{1}{Q_{x}^{2}+Q_{y}^{2}+k_{\mathrm{g}}^{2}}
$$

where $k_{\mathrm{g}}^{2}=\rho g / \gamma \approx\left(0.36 \mathrm{~cm}^{-1}\right)^{2}$ and $Q_{\max }$ is an upper cut-off that is necessary in order to fix the number of thermal surface modes. In analogy with the Debye theory of heat capacity one might guess $Q_{\max } \sim \pi /$ molecule radius $=(\pi / 1.93 \AA)$ for water. Taking $\gamma \approx 73 \mathrm{dyn}^{-1}$ this integrates to $\left\langle\eta(x, y)^{2}\right\rangle=(3.98 \AA)^{2}$ or nearly twice the measured slope.

The origin of this discrepancy is that for a finite-size detector slit the spectrometer is unable to distinguish between 'true specular reflection' and the sum of specular reflection and diffuse scattering at small angle to the specular reflection. Stated another way, for a rough surface $R(\theta)$ is less than $R_{\mathrm{F}}(\theta)$ because of destructive interference between signals reflected from different heights $\eta(x, y) \neq \eta\left(x^{\prime}, y^{\prime}\right)$. Since a spectrometer with finite resolution cannot detect interference between points that are too far apart, long-wavelength height variations do not affect the measured reflectivity. The measured slope should actually be compared to

$$
\sigma_{c}^{2} \equiv \frac{k_{\mathrm{B}} T}{4 \pi^{2} \gamma} \int_{A_{\Delta Q}}^{Q_{\max }} \mathrm{d} Q_{x} \mathrm{~d} Q_{y} \frac{1}{Q_{x}^{2}+Q_{y}^{2}+k_{\mathrm{g}}^{2}}
$$

where $A_{\Delta Q}$ is a circular area in the $Q_{x}-Q_{y}$ plane with outer radius $Q_{\max }$ and a rectangular inner cutout with dimensions determined by the spectrometer resolution, eqn $(10)$. The minimum dimensions of $A_{\Delta Q}$ are much larger than $k_{\mathrm{g}}$ and if $\Delta Q_{x} \gg \Delta Q_{y}$ the slope approximately given $b^{8}$

$$
\sigma_{c}^{2} \approx \frac{k_{\mathrm{B}} T}{2 \pi \gamma} \ln \left(\frac{2 Q_{\max }}{\Delta Q_{y}}\right) .
$$

Fig. 2(c) shows the comparison of the best fit values of $\sigma_{c}$ calculated by numerical integration of eqn (15) over the measured resolution function for data taken with three different detector heights $h .^{13}$ The only adjustable parameter in the fitting procedure was the value of $Q_{\max }=(\pi / 1.4 \AA)$ that was common to all three fits. This is slightly larger than the guess of $(\pi / 1.93 \AA)$; however, in view of the naive nature of the theory the difference is not serious. In particular X-ray measurements over the accessible range of angles cannot distinguish between one particular value of $Q_{\max }$ for an interface that is locally sharp, and a smaller value of $Q_{\max }$ for an interface that locally has a more gradual profile.

\section{Diffuse Scattering}

According to this model $R(\theta) / R_{\mathrm{F}}(\theta)<1$ because thermally excited capillary waves scatter radiation away from the specular condition. Fig. 3 illustrates diffuse scattering data from the surface of $\mathrm{H}_{2} \mathrm{O}$ that was taken by fixing the incident angle and sample position and scanning the detector angle $\theta^{\prime}$ in the plane of incidence, i.e. $\psi=0 .{ }^{13}$ The peak at $\theta=\theta^{\prime}$ is the specular signal and the weaker peaks at $\theta^{\prime} \approx \theta_{\mathrm{c}}$ correspond to the structure of $T\left(\theta^{\prime}\right)$ discussed above. The solid lines through the data are calculated by averaging the cross-section [eqn (8)] over the angular distribution of the incident beam 


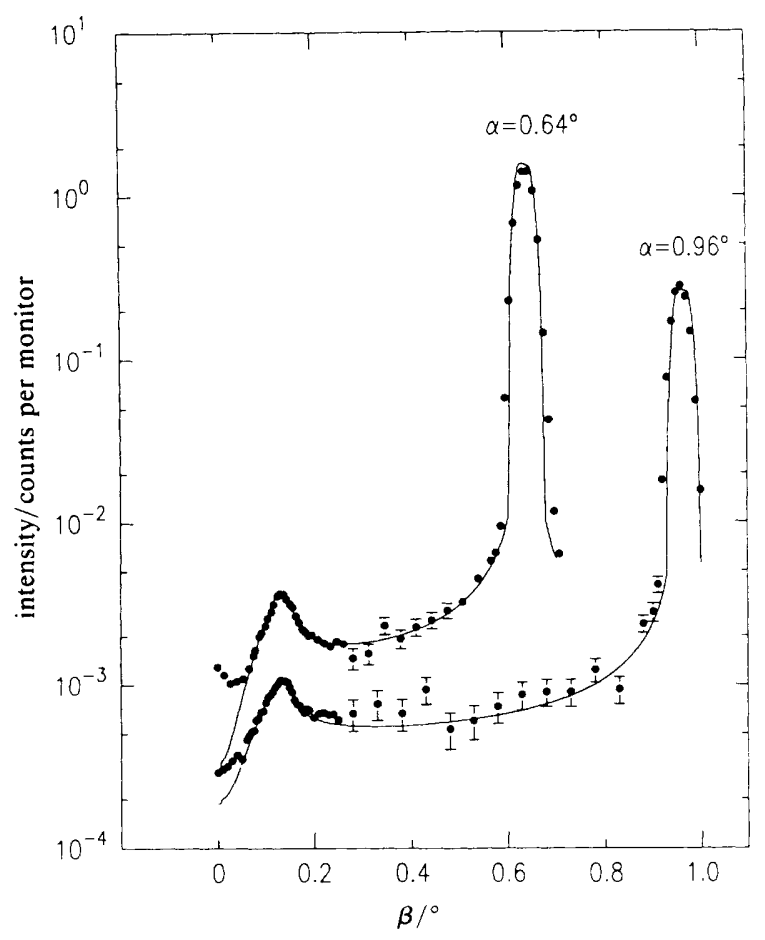

Fig. 3. Scattered intensity from the surface of $\mathrm{H}_{2} \mathrm{O}$, in the plane of incidence and as a function of detector angle $\theta^{\prime}$ for incident angles $\theta=0.64$ and $0.96^{\circ}{ }^{13}$ The peaks at $\theta^{\prime}=\theta_{c} \approx 0.13^{\circ}$ are due to the surface scattering enhancement factor, the peaks at $\theta^{\prime}=\theta$ are the specular reflectivity signals. The solid line is the theoretical prediction calculated with no significant adjustable parameters.

and integrating over the detector resolution. The only adjustable parameter is a small constant background, of the order of $10 \%$ of the peak at $\theta^{\prime}=\theta_{\mathrm{c}}$. Agreement between data and theory for both the diffuse scattering, and the resolution dependence of $R(\theta) / R_{\mathrm{F}}(\theta)$ confirms the role of thermally excited capillary waves and demonstrates the quantitative reliability of the experimental technique.

\section{Insoluble Monolayer on Water}

These two previous results for $\mathrm{H}_{2} \mathrm{O}$ buttress the hope of being able to make quantitative interpretation of $R(\theta) / R_{\mathrm{F}}(\theta)$ data on more complex surface structures. One example of this is illustrated by the data in fig. $4(a)$ for the ratio $R(\theta) / R_{\mathrm{F}}(\theta)$ of a monolayer of Lignoceric acid $\left(\mathrm{CH}_{3}\left(\mathrm{CH}_{2}\right)_{22} \mathrm{CO}_{2} \mathrm{H}\right)$ on water at $\mathrm{pH} 2$, using $\mathrm{HCl}$, at different surface pressures. ${ }^{15}$ This data, like the above data for $\mathrm{H}_{2} \mathrm{O}$ was taken on a specially constructed trough, to be described elsewhere, in which the surface tension could be continuously monitored. Fig. $4(b)$ illustrates details of the profiles that gave the best fit to the data when substituted into eqn (5). Particularly interesting is the fact that as the pressure increases the position of the local maximum moves away from the interface to the vapour, implying that the distance between the acid head group and the alkane/vapour interface has increased.

Interpretation of these specular reflectivity results can be aided by recent surface scattering studies on the in-plane structure of monolayers. A number of different groups have been applying this technique, in which the incident angle $\theta$ is adjusted to be slightly 

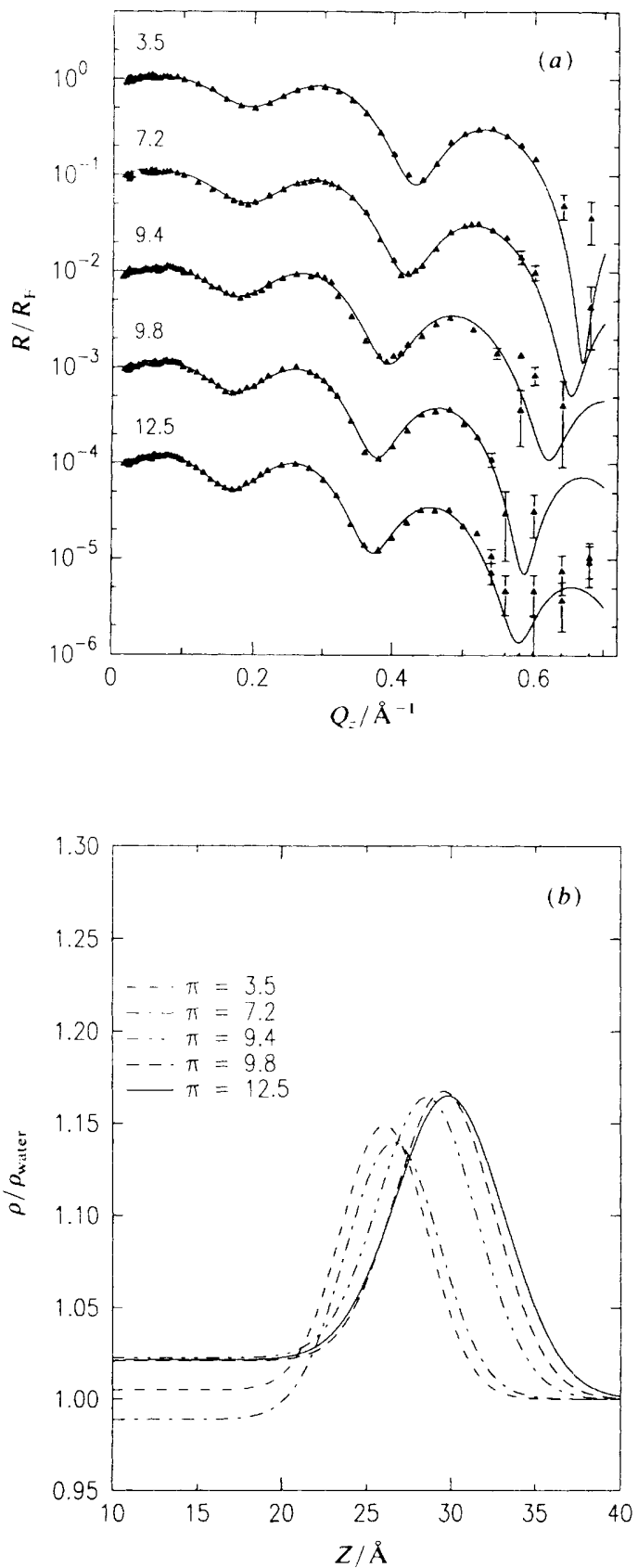

Fig. 4. (a) The ratio $R(\theta) / R_{\mathrm{F}}(\theta)$ for different surface pressures (dyn $\mathrm{cm}^{-1}$ ) of a Lignoceric acid $\left(\mathrm{CH}_{3}\left(\mathrm{CH}_{2}\right)_{22} \mathrm{CO}_{2} \mathrm{H}\right)$ monolayer on water. The solid lines correspond to the $R(\theta) / R_{\mathrm{F}}(\theta)$ predicted by best fits of real space density models. $(b)$ shows details of the electron density profiles corresponding to the best fits in $(a)$. The origin of the abscissa $(z=0)$ is defined by the alkane/vapour interface. The data were recorded at room temperature and the subphase was at $\mathrm{pH} 2$. 


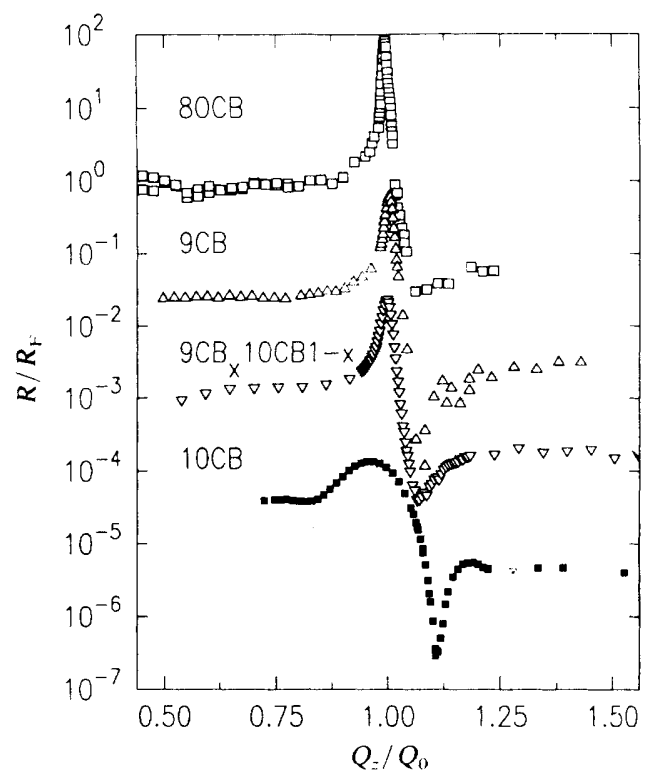

Fig. 5. The ratio of $R(\theta) / R_{\mathrm{F}}(\theta)$ for four different liquid-crystal systems: (a) 8OCB, (b) 9CB, (c) a mixture $(9 \mathrm{CB})_{1-x}(10 \mathrm{CB})_{x}$ with $x \approx 0.15$ and $(d)$ 1OCB. In all cases the temperatures are ca. $0.05^{\circ} \mathrm{C}$ above the transition to the smectic-A phase.

less than $\theta_{\mathrm{c}}$, insuring that the incident beam penetrates only evanescently into the bulk, i.e. intensity $\sim \exp (-\kappa z)$, where $\kappa \approx(2 \pi / \lambda) \sqrt{ }\left(\theta_{\mathrm{c}}^{2}-\theta^{2}\right) .{ }^{15,18-21}$ When the surface monolayer is crystalline, Bragg-like scattering from surface monolayers has been observed at angles $\psi \approx \sin ^{-1}(\lambda / 2 a)$, where $a \approx 4.3 \AA$ is the lattice spacing of the twodimensional surface crystal. By monitoring the scattered intensity as a function of the $\theta^{\prime}$ it is possible to demonstrate the existence of surface phases in which the orientation of the alkane chains with respect to the surface normal changes. These results, clearly that most of the structural features of crystalline surface monolayers can profitably be studied using X-ray techniques.

\section{Liquid Crystals}

The two examples discussed thus far dealt with systems in which the surface structure is confined to distances no more than one or two molecular lengths from the interface. Liquid crystals represent a class of systems for which the surface can induce structure that penetrates hundreds of molecular lengths into the bulk. Fig. 5 displays data showing the ratio $R(\theta) / R_{\mathrm{F}}(\theta)$ as a function of $Q_{z} / Q_{0}$, where $Q_{0}=(2 \pi / D)$ and $D$ is the smectic-A layer spacing for the respective molecules, octyloxycyanobiphenyl 8OCB, nonylcyanobiphenyl $9 \mathrm{CB}$, a mixture $(9 \mathrm{CB})_{1-x}(10 \mathrm{CB})_{x}$ with $x \approx 0.15$ and 1 OCB. In all cases the temperature is ca. $0.05^{\circ} \mathrm{C}$ above the transition to the smectic-A phase. Both $8 \mathrm{OCB}$ and $9 \mathrm{CB}$ have second-order phase transitions from the nematic to smectic-A phases, in which critical smectic fluctuations in the nematic phase have characteristic lengths along the layer normal $\left(\xi_{\| /}\right)$and parallel to the layers $\left(\xi_{\perp}\right)$ that diverge as the transition is approached; e.g. $\xi_{\|}, \xi_{\perp} \sim\left(T-T_{\mathrm{NA}} / T_{\mathrm{NA}}\right)-\nu_{\|, \perp}$ where $T_{\mathrm{NA}}$ is the nematic to smectic-A transition temperature. Analysis of the temperature dependence of the shapes of the peaks in $R(\theta) / R_{\mathrm{F}}(\theta)$ at $Q_{z} \approx Q_{0}$ establish that surface induces smectic order in the nematic phase, and that this order penetrates into the bulk a distance that is equal to 

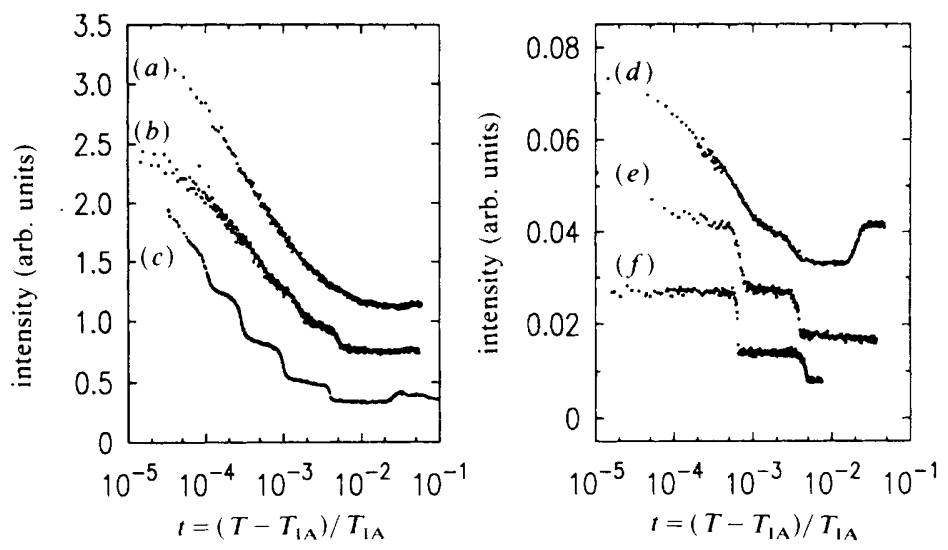

Fig. 6. Specular reflectivity as a function of temperature for $Q_{z} \approx(2 \pi / D)$ for: $(a) 10 \mathrm{CB},(b)$ $11 \mathrm{CB},(c) 12 \mathrm{CB},(d) 12 \mathrm{OCB},(e) 140 \mathrm{CB}$ and $(f) 16 \mathrm{OCB}$.

$\xi_{\| /}{ }^{22-24}$ The physical significance of this is that although the symmetry of the surface forces local smectic order, the penetration into the bulk is determined by the bulk susceptibility. This type of behaviour, in which the thickness of the surface induced phase diverges in proportion to the critical divergence of the bulk correlation length is termed critical absorption. ${ }^{25,26}$

The situation is slightly different for the mixture of $(9 \mathrm{CB})_{0.85}(10 \mathrm{CB})_{0.15}$ which has a first-order transition to the smectic-A phase. Analysis of this system indicates that the surface-induced order penetrates into the bulk from 2 to 4 times further than the bulk critical length $\xi_{\| /}$depending on the temperature. ${ }^{12,27}$ Since the transition from the nematic to smectic-A phase is first-order the critical length $\xi_{\|}$does not diverge and the 'wetting' of the surface by the smectic-A phase is incomplete. ${ }^{25,26}$ The wetting for $x=0.15$ is larger than the wetting for a mixture with $x=0.30$ and we suspect that there is a true wetting transition as $x$ approaches the tricritical point at $x \approx 0$ for $(9 \mathrm{CB})_{1-x}(10 \mathrm{CB})_{x}$ mixtures.

The case of $10 \mathrm{CB}$ is different in that that system undergoes a first-order transition from the isotropic to smectic-A phases and there is no evidence for critical smectic fluctuations in the isotropic phase. The data clearly indicate that even in the isotropic phase the surface has induced smectic order. The extent of this order, and the manner in which it develops with temperature is illustrated by the data in fig. 6 , which displays the reflected intensity at $Q_{z} \approx Q_{0}$ as a function of $t \equiv\left(T-T_{\mathrm{IA}}\right) / T_{\mathrm{IA}}$, where $T_{\mathrm{IA}}$ is the isotropic to smectic-A transition temperature, for six different liquid crystals. ${ }^{12,27,28}$ Aralysis of the angular dependence of $R(\theta) / R_{\mathrm{F}}(\theta)$ confirms that each step corresponds to an increase in the number of smectic layers. On cooling $12 \mathrm{CB}$ there is a surface transition to one layer at a reduced temperature $t=0.04$ or $T-T_{\mathrm{IA}} \approx 13{ }^{\circ} \mathrm{C}$ followed by a successive transition up to $c a$. six layers, after which the growth appears continuous. The evolution for $11 \mathrm{CB}$ has fewer discrete transitions, and the evolution for $10 \mathrm{CB}$ appears continuous. There are two important things to note regarding these data. First, mixtures in which the concentration $9 \mathrm{CB}$ is equal, or greater than that of $10 \mathrm{CB}$, have a small temperature region of nematic phase between the isotropic and smectic- $A$ that shrinks to zero for $(9 \mathrm{CB})_{1-x}(10 \mathrm{CB})_{x}$ with $x \approx 0.45 .^{29}$ Thus $10 \mathrm{CB}$ is relatively near to a region of the phase diagram in which the nematic order is stable, while $11 \mathrm{CB}$ and $12 \mathrm{CB}$ are more distant. The differences in the temperature evolution of 10,11 and $12 \mathrm{CB}$, with relatively sharp layer transitions more prominent for the longer homologues, suggest that the width of the physical interface between the surface induced smectic region and 
the bulk isotropic is broadened out, or made more diffuse by the proximity of the nematic phase. According to this interpretation the proximity of the nematic phase stabilizes a region, between the surface smectic and the bulk isotropic, that has well developed molecular orientational order but only partial smectic order. ${ }^{30,31}$ With increasing distance from the part of the phase diagram in which the nematic order is stable, as in going from $10 \mathrm{CB}$ to $11 \mathrm{CB}$ and $12 \mathrm{CB}$, this effect becomes weaker and the profile from smectic to isotropic becomes sharper. A similar effect is shown for three homologues in the $n$ OCB series; 12OCB $(d), 140 C B(e)$ and 16OCB $(f)$.

A second interesting feature of these data is that although one might argue from the data in fig. $6(a)-(c)$ that in the $n C B$ series the thickness of the surface smectic layer diverges with decreased reduced temperature, indicative of complete wetting of the isotropic surface by the smectic-A phase, it is absolutely clear that the wetting by $16 \mathrm{OCB}$ is not complete. ${ }^{\dagger}$

The surfaces of these systems exhibit an interplay between the surface-induced nematic, or molecular orientational order, and the smectic-A, or positional order. In spite of considerable effort the critical properties of the second-order transition from the nematic to smectic-A phases in the bulk are not understood and one line of speculation attributes this to an incomplete understanding of the interplay between these two order parameters. $^{32,33}$ Hopefully, the surface problem will present new insights that might guide theoretical development of theories for the bulk transition.

\section{Lyotropic}

One other type of liquid/vapour interface that we have studied is that of a micellar mixture of caesium perfluoro-octanoate(CsPFO and water. ${ }^{34}$ For a narrow range of temperatures these mixtures form lyotropic liquid crystals in which oblate micelles orient to produce a uniaxial nematic phase. As the temperature is lowered the system undergoes what appears to be a second-order transition to a smectic-A phase; however, the question of whether the smectic-A phase consists of layers of oblate micelles, or whether it consists of bilayers of amphiphillic molecules separated by layers of water is not yet settled. ${ }^{34,35}$ Fig. $7(a)$ shows data for $R(\theta) / R_{\mathrm{F}}(\theta)$ for a mixture containing $c a$. $60 \mathrm{wt} \%$ of water at temperatures 2 and $7^{\circ} \mathrm{C}$ above $T_{\mathrm{NA}}$. The first of these is in the nematic phase and the second is in the isotropic; however, they both have peaks at $Q_{z} \approx 0.117 \AA$ that correspond to $2 \pi /(54 \AA)$, where $54 \AA$ is approximately equal to the layer spacing in the bulk smectic phase. Furthermore, in both cases the peaks have lineshapes with a pronounced minimum at the low-angle side. There is not sufficient space to present a full analysis of these lineshapes; however, the principal conclusion can be illustrated by the model density profile $\langle\rho(z)\rangle$, shown in fig. $7(b)$, that was used to calculate the solid line running through the $2^{\circ} \mathrm{C}$ data in $(a) .{ }^{36}$

First, the electron density oscillates between a maximum value that is $c a .85 \%$ of the electron density of bulk fluorocarbon (i.e. $1.81 \times$ electron density of water) and the electron density of water. The full width of the surface layer is very close to the $12.5 \AA$ that corresponds to the length of the fully extended CsPFO molecule and the full width of the electron density maxima below the surface are about twice that width, or $25.0 \AA$. Taken together these indicate that surface consists of a relatively dense monolayer of CsPFO, followed by CsPFO bilayers, all of which are separated by layers of water. Since the maximum density of the subsurface bilayers is the same as the maximum density of the surface monolayer we believe that they are most likely intact bilayers, and not layers of positionally correlated micelles. While this does not exclude the possibility that the bulk smectic consists of positionally correlated micelles, we think that is unlikely.

$\dagger$ Ocko et al. ${ }^{28}$ argued that the wetting for $12 \mathrm{CB}$ was incomplete on the basis of more detailed lineshape analysis for $T \Rightarrow T_{1 \wedge}$. 

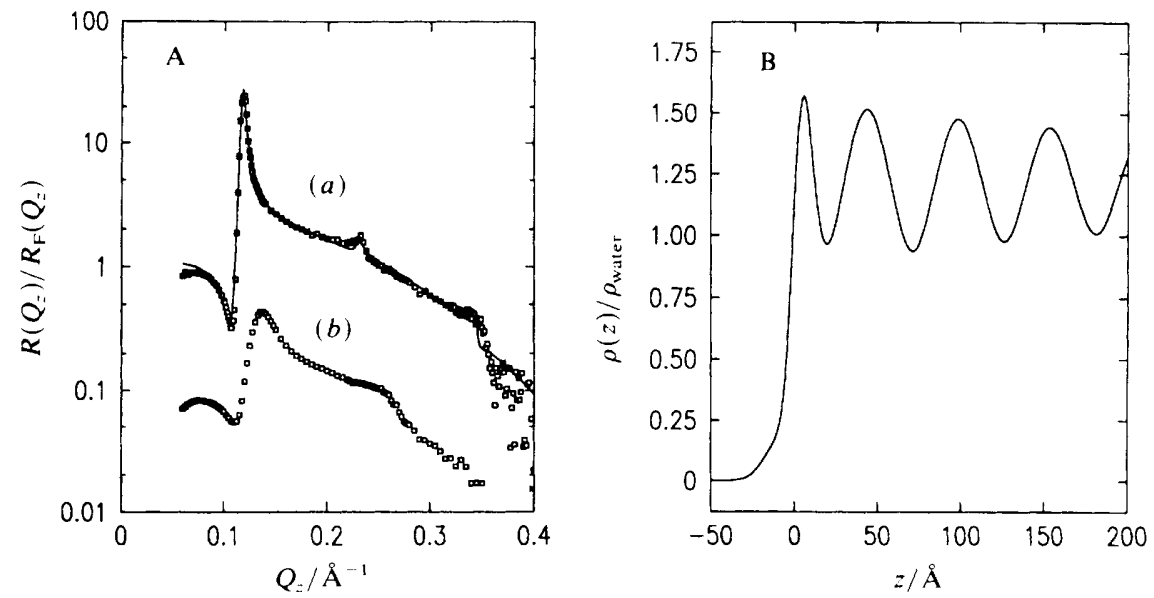

Fig. 7. $R(\theta) / R_{\mathrm{F}}(\theta)$ from the surface of a mixture of $\mathrm{CsPFO}$ and $\mathrm{H}_{2} \mathrm{O}\left(c a .60 \mathrm{wt} \% \mathrm{H}_{2} \mathrm{O}\right)$ in the nematic phase at $T-T_{\mathrm{NA}} \approx 2{ }^{\circ} \mathrm{C}$ and in the isotropic phase at $T-T_{\mathrm{NA}} \approx 7^{\circ} \mathrm{C} .(a) T-T_{\mathrm{NA}} \approx 2^{\circ} \mathrm{C}$, (b) $T-T \approx 7^{\circ} \mathrm{C}$. The solid line through the nematic data is the ratio $R(\theta) / R_{\mathrm{F}}(\theta)$ predicted from the electron density model illustrated in B.

Secondly, the similarity between the shapes of the two peaks, at $T-T_{\mathrm{NA}} \approx 2{ }^{\circ} \mathrm{C}$ in the nematic phase, and $T-T_{\mathrm{NA}} \approx 7^{\circ} \mathrm{C}$ above $T_{\mathrm{NA}}$ in the isotropic phase, together with further data not included here indicates that although the near surface region is relatively insensitive to the equilibrium bulk phase, even in the isotropic phase the surface induced order extends a number of layers below the surface. This penetration is temperature dependent, and as $T_{\mathrm{NA}}$ is approached other data not shown here indicate penetration to distances at least 100 layers (i.e. $5000 \AA$ ). The fact that the $2{ }^{\circ} \mathrm{C}$ peak is sharper, and more intense, is a consequence of this. The temperature dependence of these surface peaks provide a strong indication that the bulk nematic to smectic-A transition is truly second-order.

\section{Liquid ${ }^{4} \mathrm{He}$}

The last example of liquid surfaces to be described is illustrated by preliminary results of X-ray reflectivity from the surface of liquid ${ }^{4} \mathrm{He}^{37}$ Aside from the non-trivial cryogenic problems that must be solved in order to study this interface, the fundamental difficulty has to do with the fact that the electron density of ${ }^{4} \mathrm{He}$ is low, i.e. ca. $11 \%$ of the electron density of $\mathrm{H}_{2} \mathrm{O}$. Since the reflectivity varies as the square of the electron density this means that the reflectivity from ${ }^{4} \mathrm{He} /$ vapour interface should be $c a .1 \%$ of that of the $\mathrm{H}_{2} \mathrm{O} /$ vapour interface. Even with the largest possible synchrotron intensities this weaker signal, and its accompanying small value for $\theta_{c}$, would made the experiment very difficult. To circumvent this problem we elected to study thin layers of ${ }^{4} \mathrm{He}$ physisorbed onto the surface of a flat $\mathrm{Si} / \mathrm{SiO}$ wafer. A general expression for the ratio of the reflectivity from an ${ }^{4} \mathrm{He}$ layer of thickness $D$ on a substrate to the reflectivity from an ideal substrate with the electron density of $\mathrm{Si}$ can be represented as

$$
\frac{R(\theta)}{R_{\mathrm{F}}(\theta)}=\left|\alpha\left(Q_{z}\right)+\beta\left(Q_{z}\right) \exp \left(\mathrm{i} Q_{z} D\right)\right|^{2}
$$

where $\alpha\left(Q_{z}\right)$ and $\beta\left(Q_{z}\right)$ correspond to the amplitudes of the signal reflected from the ${ }^{4} \mathrm{He} /$ vapour interface and the ${ }^{4} \mathrm{He} / \mathrm{Si}$ interface. At small angles $\alpha(0) \approx\left(\rho^{\mathrm{He}} / \rho^{\mathrm{Si}}\right)$ and 


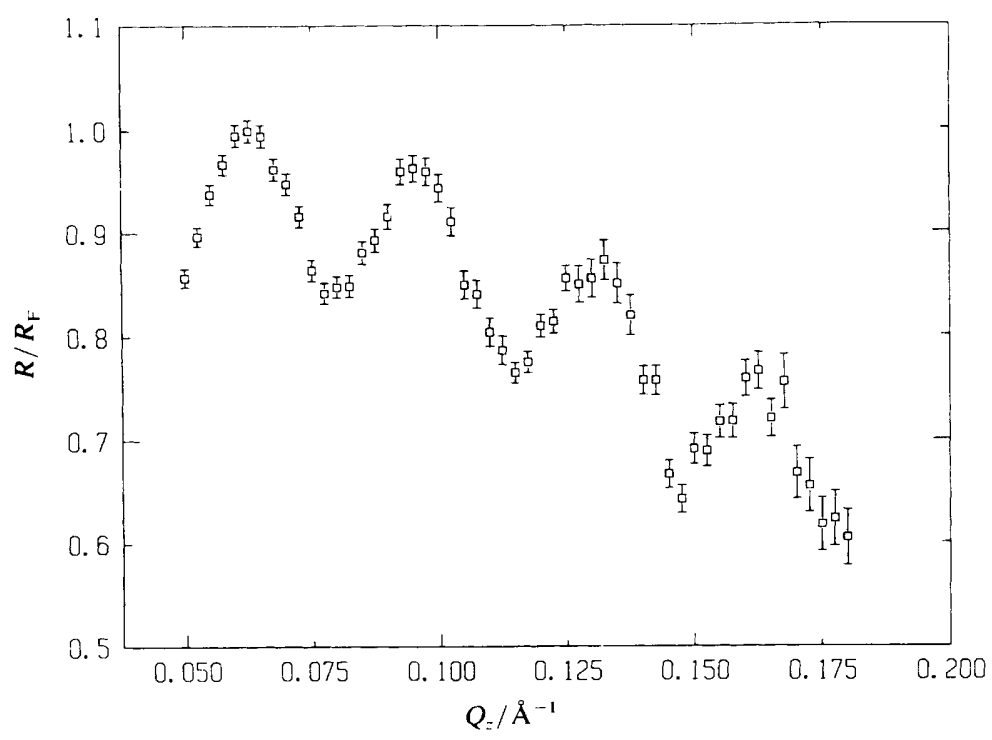

Fig. 8. Preliminary measurements of $R(\theta) / R_{\mathrm{F}}(\theta)$ for a $190 \AA$ layer of ${ }^{4} \mathrm{He}$ adsorbed on a flat wafer of $\mathrm{Si} / \mathrm{SiO}$ at $2.35 \mathrm{~K}$.

$\beta(0) \approx 1-\alpha(0)$, such that with $\alpha(0) \approx 0.05$ the cross-term $2 \alpha \beta \cos \left(Q_{z} D\right) \approx 0.1$. The predicted reflectivity oscillates with a period of $\Delta Q_{z}=2 \pi / D$ and a peak-to-peak amplitude that is $c a .20 \%$ of the mean reflectivity. This is a relatively large effect and easy to observe. Fig. 8 shows preliminary data from a $190 \AA$ thick layer at a temperature of $2.35 \mathrm{~K}$ that was measured using a $12 \mathrm{~kW}$ rotating anode $\mathrm{X}$-ray source. Both the amplitude and the period of the oscillations are within a few percent of the theoretically expected values. The overall decay with increasing $Q_{z}$ reflects the roughness of the bare $\mathrm{Si} / \mathrm{SiO}$ substrate. More specifically, since both the ${ }^{4} \mathrm{He} /$ vapour and the ${ }^{4} \mathrm{He} / \mathrm{Si}$ interfaces have finite widths, both $\alpha\left(Q_{z}\right)$ and $\beta\left(Q_{z}\right)$ must decrease with increasing $Q_{z}$. Using synchrotron radiation it should be able to follow the interference oscillations over at least four times as many periods as for this data set. From the variation of their amplitudes with angle we expect to determine the width of the ${ }^{4} \mathrm{He} /$ vapour interface as a function of both temperature and film thickness. This is a problem that has received very much theoretical attention. $^{38}$

\section{Summary}

The main goal of this paper has been to present the underlying concepts behind the use of X-ray specular reflectivity to study liquid surfaces. In fact, these same ideas carry over to the study of solid surfaces and, in some cases, such as for the study of buried solid-solid interfaces, $\mathrm{X}$-ray reflectivity may facilitate measurements that are not practical by other techniques. ${ }^{16,39,40}$ The microscopic structure of liquid surfaces, on the other hand, cannot be studied by very many techniques, and we have tried to illustrate by example some of the types of measurements that can, and have been done. In almost all cases neutron scattering can be used in much the same way as X-rays to carry out similar studies; however, there are two main differences. ${ }^{41}$ First, $X$-rays have the advantage that synchrotron sources provide many orders of magnitude larger incident flux, per solid angle than any conceivable neutron source. As a consequence, specular reflectivity of $\mathrm{X}$-rays can be carried out over dynamic ranges of the order of $10^{10}$ or 
larger, while for neutrons it is difficult to achieve a dynamic range of $10^{6}$. Since the spatial resolution of the reflectivity technique is directly related to the attainable range of $Q_{z}$ and since the reflectivity falls rapidly with increasing $Q_{z}$ this is a severe limitation on use of neutrons for certain classes of problems.

The main advantage of neutrons derive from the fact that by substitution of deuterium for hydrogen it is possible to vary the contrast between different parts of organic fluids. For example, it is very difficult to do precise small-angle reflectivity studies of polymer conformation at the liquid/vapour interface for the purpose of characterizing the power-law dependence of the polymer density at large distances. ${ }^{42-44}$ The difficulty arises primarily because the critical angle $\theta_{c}$, typically of the order of $0.15^{\circ}$, corresponds to a $Q_{z} \approx 0.02 \AA^{-1}$ in the vapour. As $\theta \Rightarrow \theta_{\text {c }}$ refraction effects result in smaller values of $Q_{z}$ inside the material; however, since these are delicately dependent on both the direction of the incident beam and the orientation of the surface normal it is difficult to make quantitatively accurate measurements at values of $Q_{z}$ inside the material that are significantly smaller than $(4 \pi / \lambda) \theta_{c}$. For neutrons, on the other hand, with a suitable mixture of protons and deuterons the value of $\theta_{c}$ can be reduced to zero, and it is relatively easy to measure the specular reflectivity at angles that are much less than $0.15^{\circ}$.

In general, specular reflection using both neutron and $\mathrm{X}$-rays are promising techniques for the study of liquid surfaces. They each have specific advantages and in many cases they compliment each other. The structure of the interface between a polymer solution and its vapour is just one example of a problem for which full understanding will surely require both types of measurements.

This work was supported by the National Science Foundation through grants to the Harvard Materials Research Laboratory, NSF-DMR-88-12855 and NSF-DMR-86-14003. Research carried out at the NSLS, Brookhaven National Laboratory, is supported by the Department of Energy, Material Sciences and Division of Chemical Sciences under contract DE-AC02-76CH00016.

\section{References}

1 J. A. Prins, Z. Phys., 1928, 47, 479.

2 E. Nahrig, Phys. Z., 1930, 31, 401.

3 H. Kiessig, Ann. Phys., 1931, 10, 769.

4 A. H. Compton and S. K. Allison, X-Rays in Theory and Experimentation (Van Nostrand, New York, 1935).

5 See the following brief review and the references therein: S. A. Rice, Nature (London), 1985, $316,108$.

6 P. S. Pershan and J. Als-Nielsen, Phys. Rev. Lett., 1984, 52, 759.

7 J. Als-Nielsen, Solid and Liquid Surfaces Studied by Synchrotron X-Ray Diffraction in Structure and Dynamics of Surfaces II: Topics in Current Physics, ed. W. Schommers and P. van Blanckenhagen (Springer-Verlag, Berlin, 1987), vol. 43, p. 181.

8 A. Braslau, P. S. Pershan, G. Swislow, B. M. Ocko and J. Als-Nielsen, Phys. Rev. A, 1988, $38,2457$.

9 S. K. Sinha, E. B. Sirota, S. Garoff and H. B. Stanley, Phys. Rev. B, 1988, 38, 2297.

10 R. S. Becker, J. A. Golovchenko and J. R. Patel, Phys. Rev. Lett., 1986, 50, 153.

11 J. Als-Nielsen and P. S. Pershan, Nucl. Instrum. Methods, 1983, 208, 545.

12 P. S. Pershan, J. Phys. (Paris), 1989, 50, C7-1.

13 D. K. Schwartz, M. L. Schlossman, E. H. Kawamoto, G. J. Kellogg, P. S. Pershan and B. M. Ocko, Phys. Rev. A, 1990, in the press.

14 J. Daillant, L. Bosio, J. J. Benattar and J. Meunier, Europhys. Lett., 1989, 8, 453.

15 M. L. Schlossman, D. K. Schwartz, E. Kawamoto, G. J. Kellogg and P. S. Pershan, X-ray Reflectivity of a Long Chain Fatty Acid Monolayer at the Water/Vapor Interface, Materials Research Society Meeting, Boston, MA, Nov. 1989.

16 1. M. Tidswell, B. M. Ocko, P. S. Pershan, S. R. Wasserman, G. M. Whitesides and J. D. Axe, Phys. Rev. B, 1990, 41, 1111.

17 A. Braslau, M. Deutsch, P. S. Pershan, A. H. Weiss, J. Als-Nielsen and J. Bohr, Phys. Rev. Lett., 1985, 54, 114.

18 S. G. Wolf, E. M. Landau, M. Lahav, L. Leiserowitz, M. Deutsch, K. Kjaer and J. Als-Nielsen. Thin Solid Films, 1988, 159, 29. 


\section{P. S. Pershan}

19 S. G. Wolf, L. Leiserowitz, M. Lahav, M. Deutsch, K. Kjaer and J. Als-Nielsen, Nature (London), $1987,328,63$.

20 P. Dutta, J. B. Peng, B. Lin, J. B. Ketterson, M. Prakash, P. Georgopoulos and S. Ehrlich, Phys. Rev. Lett., 1987, 58, 2228.

21 P. Dutta, K. Halperin, J. B. Ketterson, J. B. Peng, G. Schaps and J. P. Baker, Thin Solid Films, 1985, 134,5 .

22 J. Als-Nielsen, F. Christensen and P. S. Pershan, Phys. Rev. Lett., 1982, 48, 1107.

23 P. S. Pershan and J. Als-Nielsen, Phys. Rev. Lett., 1984, 52, 759.

24 P. S. Pershan, A. Braslau, A. H. Weiss and J. Als-Nielsen, Phys. Rev., 1987, 35, 4800.

25 M. E. Fisher, J. Chem. Soc., Faraday Trans. 1, 1986, 82, 1569.

26 P. G. de Gennes, Rev. of Mod. Phys., 1985, 57, 827.

27 A. Braslau, Thesis (Harvard University, Cambridge, MA., 1988).

28 B. M. Ocko, A. Braslau, P. S. Pershan, J. Als-Nielsen and M. Deutsch, Phys. Rev. Lett., 1986, $57,94$.

29 J. Thoen, H. Marynissen and W. Van Dael, Phys. Rev. Lett., 1984, 52, 204.

30 J. V. Selinger, Phys. Rev. A, 1988, 37, 1736.

31 A. A. Chernov and L. V. Mikheev, Phys. Rev. Lett., 1988, 60, 2488.

32 T. C. Lubensky, J. Chem. Phys., 1983, 80, 31.

33 C. Dasgupta, Phys. Rev. Lett., 1985, 55, 1771.

34 N. Boden and M. C. Holmes, Chem. Phys. Lett., 1984, 109, 76.

35 M. C. Holmes and J. Charvolin, J. Phys. Chem., 1984, 88, 810.

36 G. Swislow, D. Schwartz, P. S. Pershan, B. Ocko, D. Litster and A. Braslau, to be published.

37 L. Lurio, T. Rabedeau, I. Silvera and P. S. Pershan, to be published.

38 D. O. Edwards, Physica, 1982, 109 \& 110B, 1531.

39 S. M. Heald, H. Chen and J. M. Tranquada, Mater. Res. Soc. Symp. Proc., 1986, 54, 165.

40 R. A. Cowley and T. W. Ryan, J. Phys. D, 1987, 20, 61.

41 See, for example, the several papers in the Proceedings of the International Conference on Surface and Thin Film studies using Glancing-Incidence X-ray and Neutron Scattering, Marseille (France, 1989), ed. M. Bienfait and J. M. Gay; J. Phys. (Paris), 1989, 50, Colloq. 7.

42 R. Vilanove and F. Rondelez, Phys. Rev. Lett., 1980, 45, 1502.

43 D. Ausserre, H. Hervet and F. Rondelez, Phys. Rev. Lett., 1985, 54, 1948.

44 J. M. Bloch, M. Sansone, F. Rondelez, D. G. Peiffer, P. Pincus, M. W. Kim and P. M. Eisenberger, Phys. Rev. Lett., 1985, 54, 1039. 